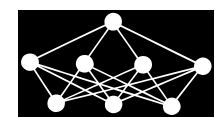

\title{
MULTI-STEP HYBRID PREDICTION MODEL OF BALTIC SUPERMAX INDEX BASED ON SUPPORT VECTOR MACHINE
}

\author{
F. Guan ${ }^{* \dagger}$, Z. Peng*t ${ }^{* \dagger}$ K. Wang ${ }^{\dagger}$ X. Song ${ }^{\dagger}$, J. Gao ${ }^{\ddagger}$
}

\begin{abstract}
Accurate prediction of the Baltic index makes great difference to the strategic decision and risk avoidance of the enterprise. For the multi-step Baltic Supermax Index prediction, direct prediction and iterative prediction has its own advantages. Therefore, in this paper, in combination with direct and iterative prediction, based on Support Vector Machine (SVM), a hybrid multistep prediction model is put forward. In hybrid model, the output from the iterative model is a rough prediction and it need also be adjusted based on the output from the direct model. And weekly BSI data from January 2011 to November 2014 are used to test the model. The results show that the hybrid multistep prediction model based on SVM has high accuracy, and is feasible in the BSI prediction.
\end{abstract}

Key words: BSI prediction, Support Vector Machine (SVM), multi-step, hybrid

Received: April 29, 2015

DOI: $10.14311 /$ NNW.2016.26.012

Revised and accepted: October 12, 2015

\section{Introduction}

Nowadays, more than $90 \%$ of international trade is done through shipping. As an important component of international shipping market, the dry bulk shipping market occupies the big half of the total global seaborne, and plays an important role in the shipping market. Due to the influence of many factors such as political, economic and natural conditions, the international dry bulk market fluctuates dramatic, which is called as one of the transportation markets that have the largest risk. The dry bulk freight indexes represent the freight rate levels of dry bulk shipping market. It not only reflects the volatility of dry bulk shipping market, but also can reflect the development status of the global economy and the trend of international trade. So it is called as the "barometer" of dry bulk shipping market. It is because of this characteristic, many insiders and experts have tried to estimate

\footnotetext{
*Feng Guan, Zixuan Peng, Innovation Center for Transport Studies, Dalian Maritime University, Dalian,116026, P.R.China, E-mail: guanfeng_dl@163.com, Pengzx_dl@163.com

$\dagger$ Keming Wang, Xiaolin Song, Feng Guan, Zixuan Peng, Transportation Management College, Dalian Maritime University, Dalian, 116026, PR China, E-mail: wangkm_7788@163.com, songxl_dl@163.com

$\ddagger$ Junjie Gao - Corresponding author, School of Automotive Engineering, Dalian University of Technology, Dalian, 116024, China, E-mail: gaojunjie@dlut.edu.cn
} 
the future trend of dry bulk shipping market through accurate forecast of the bulk freight index to guide the enterprise strategic decision so as to avoid market risk.

So far, the research about freight index prediction at home and abroad mainly includes the following contents. Kavussanos (1996) used GARCH models esent volatility in shipping markets as a time-varying process [9]. In 1997, Berg-Andreassen selected freight rates of 10 routes from April 1985 to December 1985, and used Dickey-Fuller test methods to give a detailed analysis of the trend of Baltic dry freight index. The results showed that the selected dry bulk freight rates all obey random walk sequence [2]. Veenstra and Franses used econometric method to forecast index. By means of index time series with the process of product and unit root test method, they respectively established the first-order vector autoregressive model (VAR) on the shipping freight index time series of different dry bulk ship types and routes [17]. Cullinane, Mason and Cape accurately forecasted the spot rate through ARMA model of simple single variable [3]. Li and Parsons predicted oil tankers transport rate by neural network. Compared with the ARMA model, it was proved that for a relatively long time series model, neural network model has higher prediction accuracy [12]. British scholar Kavussanos and Alizadeh-M created a seasonal autoregressive integral moving average model of single variable and a vector autoregressive model (VAR) to study index and analyze the seasonal characteristics of dry bulk shipping market, and seasonal fluctuation characteristics of dry bulk shipping market is concluded [7]. VECM-GARCH model was used by Kavussanos and Visvikis to investigate the lead-lag relationship in both return and volatilities between spot and future markets [8]. Using a hybrid model of wavelets and neural networks, Leonov and Nikolov studied fluctuations in the freight rates of the Baltic Panamax route 2A and the Baltic Panamax route 3A. The wavelet multiscale decomposition of time series reveals volatility dynamics across different time frequencies and will uncover patterns that will be used by neural networks for prediction [11]. Fan et al analyzed the statistical data of the Baltic Capesize Freight Index (BCI) and the daily return rate sequences to improve forecast reliability of the international dry bulk shipping market [4]. Han et al adopted wavelet transform to denoise the BDI data series and developed a combined model of wavelet transform and support vector machine to forecast BDI [5]. Zeng et al proposed a method based on empirical mode decomposition (EMD) and artificial neural networks (ANN) is developed for Baltic Dry Index (BDI) forecasting [26]. Other papers about shipping index prediction can be seen in $[6,14,1]$.

Recently, as a new machine learning method, SVM has attracted many researchers' attention. SVM was developed by Vapnik, which is characterized by a specific type of machine learning methods $[15,16]$. It has been successfully applied to solving some classic problems, such as forecasting of financial market [18], electricity price [13], forecasting of bus arrival time [21, 22, 23, 24, 25], rock displacement surrounding a tunnel [19], Freeway Incident Detection [20]. Based on collected statistics, SVM is available in small samples. Because SVM has a strong capacity for learning, it is easy to make balance on data fitting and data generalization.

However, only a few scholars use SVM to predict freight index by now. They do not get the very ideal forecast effect, and the practicability and reliability are poor. Besides, freight index prediction research mainly concentrates on the BFI 
(Baltic Freight index) or BDI (Baltic Dry Index), and the research on freight index BPI (Baltic Panalnax index), BCI (Baltic Capesize index), BHI (Baltic Handysize index), BSI (Baltic Supermax index) of single ship type is less. Therefore, this paper uses the advantage of SVM to solve the complicated nonlinear problem on shipping BSI prediction. This paper establishes the SVM hybrid multi-step prediction model, combining with the single step prediction and direct prediction. The efficiency and precision of the prediction of the model is successfully improved. This paper is organized as follows. Section 2 introduces the basic principle of support vector machine. Section 3 develops the prediction model, which includes direct prediction model, iterative prediction model and hybrid model. In Section 4, the prediction model is tested, and the performances of different model are compared and analyzed. Finally, the conclusion about this paper is presented in Section 5.

\section{Support Vector Machines for regression}

\subsection{The basic principle of Support Vector Machine}

The basic idea to estimate the regression function using the SVM model is that, through a nonlinear mapping $Y$, data $x$ of input space is mapped to a highdimensional feature space, then the linear regression is done in the high-dimensional space. In a given data set of points $G=\left\{\left(x_{i}, x_{j}\right)\right\}_{i=1}^{n}, x_{i}$ is the input vector, $y_{i}$ is the expected value, $n$ is the total number of data points. In SVM, the estimate equation is used as follows:

$$
f(x)=(w \cdot \Phi(x))+b,
$$

where $\Phi(x)$ is the nonlinear mapping from input space to high dimension feature space, and coefficients $w$ and $b$ are estimated by minimizing the Eq. (2):

$$
R_{\mathrm{reg}}(f)=C \frac{1}{n} \sum_{i-1}^{n} L_{\varepsilon}\left(y_{i}, f\left(x_{i}\right)\right)+\frac{1}{2}\|w\|^{2},
$$

where $L_{\varepsilon}$ is the loss function. This defines a $\varepsilon$ tube (Fig. 1) so that if the predicted value is within the tube the loss is zero, while if the predicted point is outside the tube, the loss is the magnitude of the difference between the predicted value and radius $\varepsilon$ of the tube. The expression of $L_{\varepsilon}$ is shown in Eq. (3).

$$
L_{\varepsilon}\left(y_{i}, f\left(x_{i}\right)\right)=\left\{\begin{array}{cl}
|y-f(x)|-\varepsilon & |y-f(x)| \geq \varepsilon, \\
0 & |y-f(x)|<\varepsilon,
\end{array}\right.
$$

where the first part $C \frac{1}{n} \sum_{i-1}^{n} L_{\varepsilon}\left(y_{i}, f\left(x_{i}\right)\right)$ is the empirical risk measured by the insensitive loss function given by Eq. (3). The second part $\frac{1}{2}\|w\|^{2}$ is regularization to make the function much smooth, so as to improve the generalization ability of estimation function. $C$ is a punishment parameter, which decides the balance between the empirical risk and the regularization part. Based on the insensitive loss function $\varepsilon$, Eq. (2) can be summed up in the following quadratic convex programming problem under convex constraint conditions as 


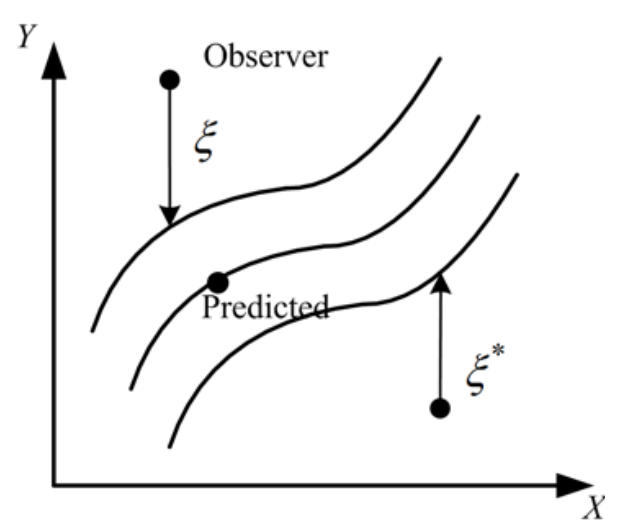

Fig. 1 The parameters for the support vector regression.

$$
\min \left\{\frac{1}{2}\|w\|^{2}+C \sum_{i-1}^{n}\left(\xi_{i}+\xi_{i}^{*}\right)\right\}
$$

s.t.

$$
\left\{\begin{array}{c}
w \Phi\left(x_{i}\right)+b-y_{i} \leq \varepsilon+\xi_{i}^{*} \\
-w \Phi\left(x_{i}\right)-b+y_{i} \leq \varepsilon+\xi_{i} \\
\xi_{i}^{*}, \xi_{i} \geq 0 \\
(i=1, \ldots, n)
\end{array}\right.
$$

where $\xi_{i}$ and $\xi_{i}^{*}$ are the introduced slack variables. The duality theory is generally adopted in solving process, which puts it into a quadratic programming problem. Through the establishment of Lagrange equation, and making $k(x, y)=\Phi(x) \cdot \Phi(y)$, the dual optimization problem can be got as

$$
\begin{gathered}
\max \left\{-\frac{1}{2} \sum_{i=1}^{n}\left(\alpha_{i}^{*}-\alpha_{i}\right)\left(\alpha_{j}^{*}-\alpha_{j}\right) K\left(x_{i}, x_{j}\right)-\varepsilon \sum_{i=1}^{n}\left(\alpha_{i}^{*}+\alpha_{i}\right)+\sum_{i=1}^{n} y_{i}\left(\alpha_{i}-\alpha_{i}^{*}\right)\right\} \\
\text { s.t. } \sum_{i=1}^{n}\left(\alpha_{i}-\alpha_{i}^{*}\right)=0, \alpha_{i}, \alpha_{i}^{*} \in[0, C],
\end{gathered}
$$

where $K\left(x_{i}, x_{j}\right)$ is the kernel function. The choice of kernel function should make it as a dot product in the feature space, namely the existence $k(x, y)=\Phi(x) \cdot \Phi(y)$. It can be proved that symmetric function $K\left(x_{i}, x_{j}\right)$ can meet the requirements of the kernel function as long as it meets the Mercer [6] conditions. Commonly used kernel functions are:

Polynomial kernel function is

$$
K\left(x_{i}, x_{j}\right)=\left(\gamma\left(x_{i} \cdot x_{j}\right)+r\right)^{d}, \gamma>0
$$

RBF kernel function is

$$
K\left(x_{i}, x_{j}\right)=\exp \left(-\gamma\left\|x_{i}-x_{j}\right\|^{2}\right), \gamma>0
$$


Sigmoid kernel function is

$$
K\left(x_{i}, x_{j}\right)=\tanh \left(\gamma\left(x_{i} \cdot x_{j}\right)+r\right) .
$$

According to the Karush Kuhn Tucker theorem, the finally regression function expression is

$$
f(x)=\sum_{i=1}^{n}\left(a_{i}-a_{i}^{*}\right) K\left(x_{i}, x\right)+b .
$$

\section{Model developments}

For prediction of BSI, the data of the next two month is the most valuable, because the accuracy prediction can provide strong support for decision making. Therefore, based on the observed data of each week, this paper intends to predict the BSI in the next two month.

Referring to multi-step prediction [10,27], there may exist two ways of BSI forecasting, iterative approaches and direct approaches. Regardless of iterative approaches or direct approaches, they are both based on basic SVM prediction model, the structure of basic SVM prediction model can be seen in Fig. 2. As we can see in it, the input vectors are the BSI of former $n$ weeks, i.e. $\mathrm{BSI}_{k}, \mathrm{BSI}_{k-1}, \ldots, \mathrm{BSI}_{k-n+1}$, where $k$ is the number of the current week, while the output variable is the BSI of target weeks, namely $\mathrm{BSI}_{k+t}$.

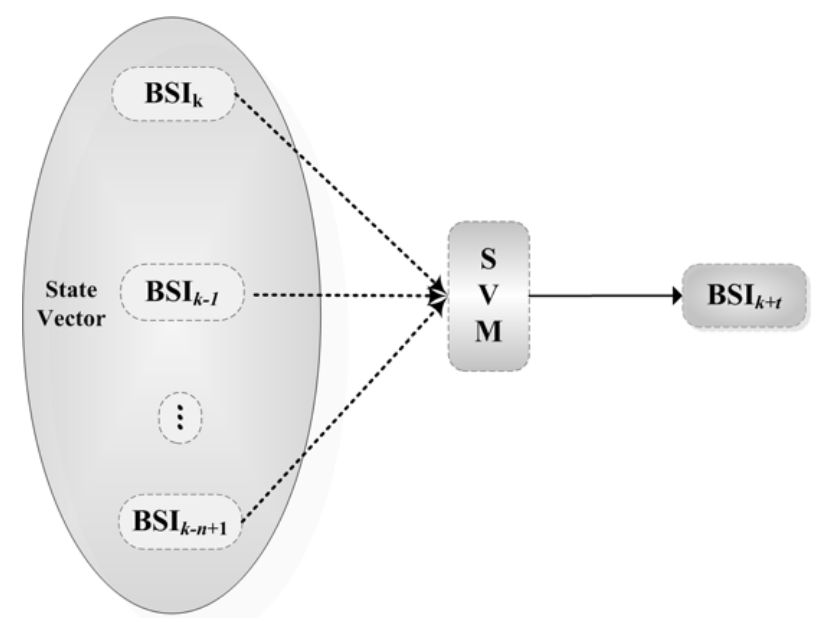

Fig. 2 Structure of basic SVM prediction model.

\subsection{Direct prediction model}

In direct prediction model, as we can see in Fig. 3, the prediction for BSI of different target week is mutually independent. And in each sub prediction, the basic SVM prediction model is used, adjusting the target week $t$ from 1 to $\gamma$. Therefore, in 
each sub prediction, the SVM model is not the same. Clearly, the prediction model can be concluded as follow:

$$
\begin{aligned}
\operatorname{SVM}(1): \mathrm{BSI}_{k+1} & =f\left(\mathrm{BSI}_{k}, \mathrm{BSI}_{k-1} \ldots \mathrm{BSI}_{k-n+1}\right) \\
\operatorname{SVM}(2): \mathrm{BSI}_{k+2} & =g\left(\mathrm{BSI}_{k}, \mathrm{BSI}_{k-1} \ldots \mathrm{BSI}_{k-n+1}\right) \\
& \ldots \\
\operatorname{SVM}(\gamma): \mathrm{BSI}_{k+\gamma} & =h\left(\mathrm{BSI}_{k}, \mathrm{BSI}_{k-1} \ldots \mathrm{BSI}_{k-n+1}\right)
\end{aligned}
$$

And, one thing we should know clearly, before prediction, we need to build the databases and train the SVM model. For each sub prediction, the database construction is different. So if you want to prediction another BSI, it needs to construct databases and train SVM model again. So, the expansibility of direct prediction is not good and should be improved.

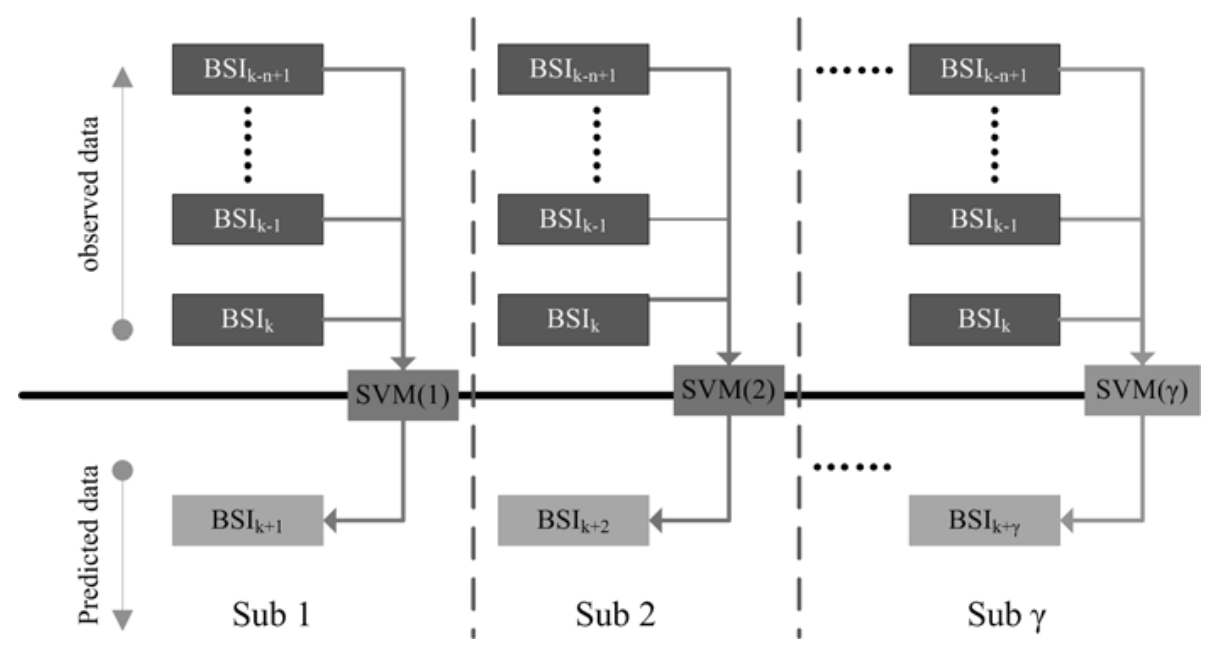

Fig. 3 Structure of direct prediction model.

\subsection{Iterative prediction model}

The iterative prediction can be decomposed into some sub prediction. In each sub prediction, the basic prediction is used, and the parameter $t$ is set as 1 . In the iterative prediction model, firstly, take the former $n$ weeks BSI, i.e. $\mathrm{BSI}_{k}, \mathrm{BSI}_{k-1}$, $\mathrm{BSI}_{k-n+1}$ as the input vectors to predict $\mathrm{BSI}_{k+1}$ by $\operatorname{SVM}(1)$. The second step is to predict $\mathrm{BSI}_{k+2}$ using $\operatorname{SVM}(2)$. Before that, we should update the input vectors, through joining $\mathrm{BSI}_{k+1}$ and eliminate $\mathrm{BSI}_{k-n+1}$, keeping the state vector number $n$ unchanged. Finally, through iterating as stated, we can predict BSI of target week $\mathrm{BSI}_{k+\gamma}$. Clearly, in iterative prediction, the SVM model is unique, and the input vectors are what changes. As Fig. 4 shows, the prediction model can be concluded as follow: 
Guan F. et al.: Multi-step hybrid prediction model of baltic...

$$
\begin{aligned}
\mathrm{BSI}_{k+1} & =f\left(\mathrm{BSI}_{k}, \mathrm{BSI}_{k-1} \ldots \mathrm{BSI}_{k-n+1}\right), \\
\mathrm{BSI}_{k+2} & =f\left(\mathrm{BSI}_{k+1}, \mathrm{BSI}_{k} \ldots \mathrm{BSI}_{k-n+2}\right), \\
& \ldots \\
\mathrm{BSI}_{k+\gamma} & =f\left(\mathrm{BSI}_{k+\gamma-1}, \mathrm{BSI}_{k+\gamma-2} \ldots \mathrm{BSI}_{k+\gamma-n}\right) .
\end{aligned}
$$

In addition, as we can see in Fig. 4, the prediction for $\mathrm{BSI}_{k+\gamma}$ include multi steps, among which the error will continued accumulated. Therefore, the prediction accuracy is usually not satisfying.

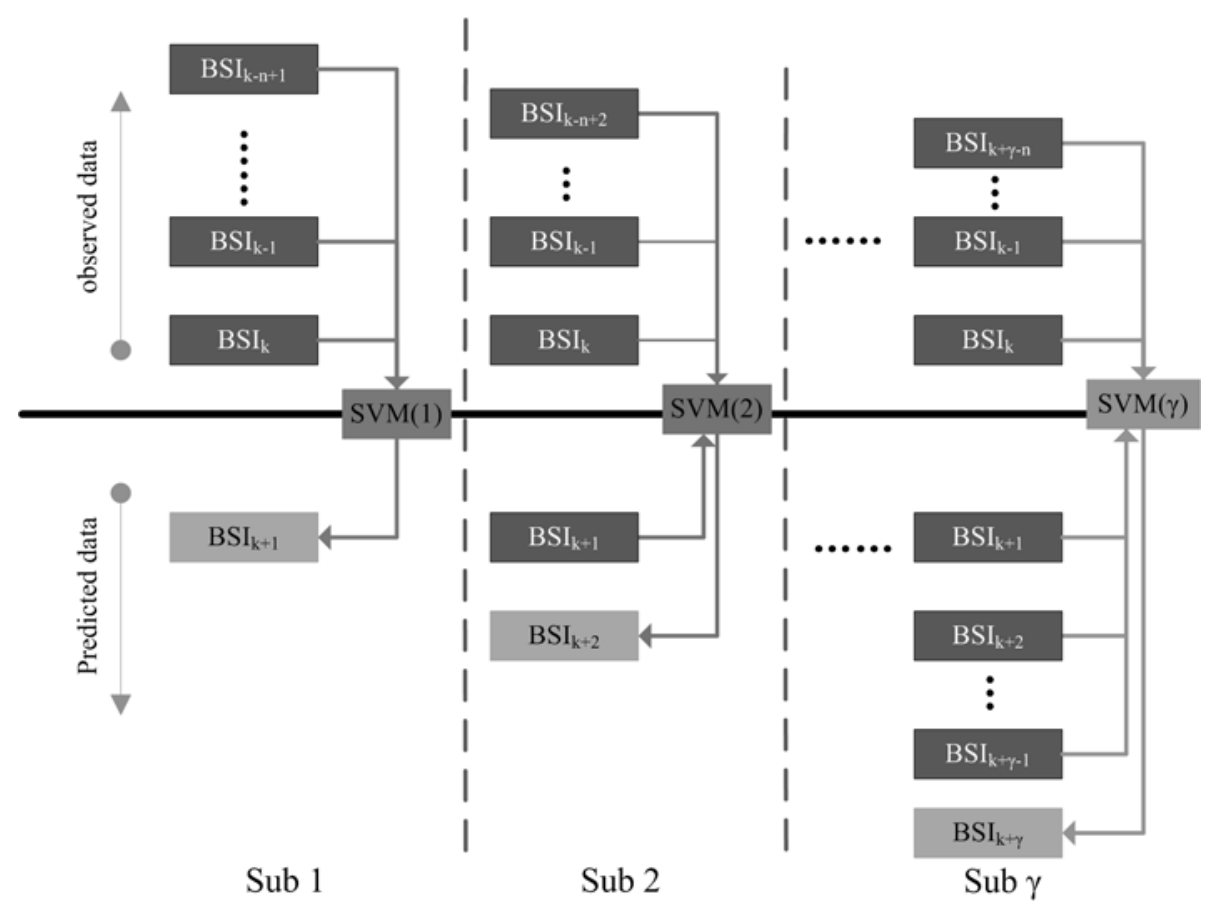

Fig. 4 Structure of iterative prediction model.

\subsection{BSI hybrid prediction model}

For the BSI multi-step forecast, direct and iterative prediction has its own advantages. Therefore, this paper combines direct prediction with iterative prediction, and proposes a BSI multi-step hybrid prediction model. Direct prediction is a single step prediction for BSI index of specified time, without iterative prediction process, so the performance is more accurate and the error is smaller. Iterative prediction model repeatedly use the single-step prediction model to directly predict the BSI index of the next week, which does not need to develop multiple SVM prediction model, but the prediction error accumulating after repeated prediction process. Therefore, by combining direct prediction model and iterative prediction 
model, the predictive values of the control points in direct prediction model can be used to adjust the predictive value between the two control points in multi-step prediction model.

As we can see in Fig. 5, assume that the first control point is the week $k$ and the second control point is the week $k+\gamma$. The prediction of BSI using either direct or iterative prediction for only one step is considered accurate. So in Fig. 5, the prediction of BSI of next week $(k+1)$ and next control point, that is week $(k+\gamma)$, are first considered to be accurate. Therefore, the adjustment operation is used to improve the BSI value after the next week $(k+1)$. The process can then be represented as follows:

$$
\widehat{\mathrm{BSI}}_{k+l}=\frac{\widehat{\mathrm{BSI}}_{k+\gamma}^{\text {direct }} \times \widehat{\mathrm{BSI}}_{k+l}^{\text {iterative }}}{\widehat{\mathrm{BSI}}_{k+\gamma}^{\text {iterative }}} \quad \gamma>l>1
$$

where $\widehat{\mathrm{BSI}}_{k+l}$ is the predicted BSI of week $k+l$ using the hybrid model. $\widehat{\mathrm{BSI}}_{k+\gamma}^{\text {direct }}$ is the predicted BSI of next control point using the direct approach. $\widehat{\mathrm{BSI}}_{k+\gamma}^{\text {iterative }}$ is the predicted BSI of next control point using the iterative approach. Here we adjust the BSI according to the proportion.

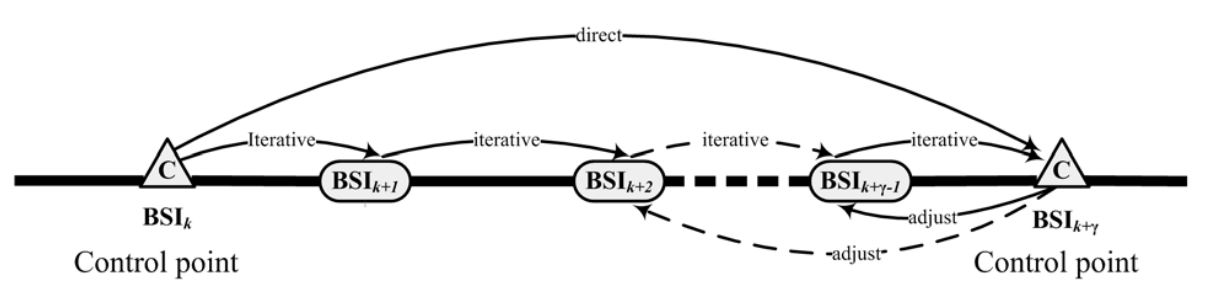

Fig. 5 Structure of hybrid prediction model.

\section{Numerical test}

\subsection{Data collection and process}

\subsubsection{Data collection}

We use weekly averaged BSI from January, 2011 and November, 2014 as simple data. The sample size is 203. The mean of the BSI weekly data is 11007.79 , the standard deviation is 2733.44. The maximum and the minimum are 16820.2 and 6487.8, respectively. The BSI data distribution can be seen in Fig. 6 .

\subsection{Data process}

To avoid the training error resulting from dimension in sample data or a large dimension data value, the whole data should be normalized and processed before the SVM training. The normalized equation is as follows: 


$$
S_{i}{ }^{\prime}=2 \cdot \frac{S_{i}-S_{\min }}{S_{\max }-S_{\min }}-1,
$$

where $S_{i}{ }^{\prime}$ is normalized value. $S_{i}$ is raw value. $S_{\min }$ is the minimum value in a sequence of samples. $S_{\max }$ is the maximum value in a sequence of samples.

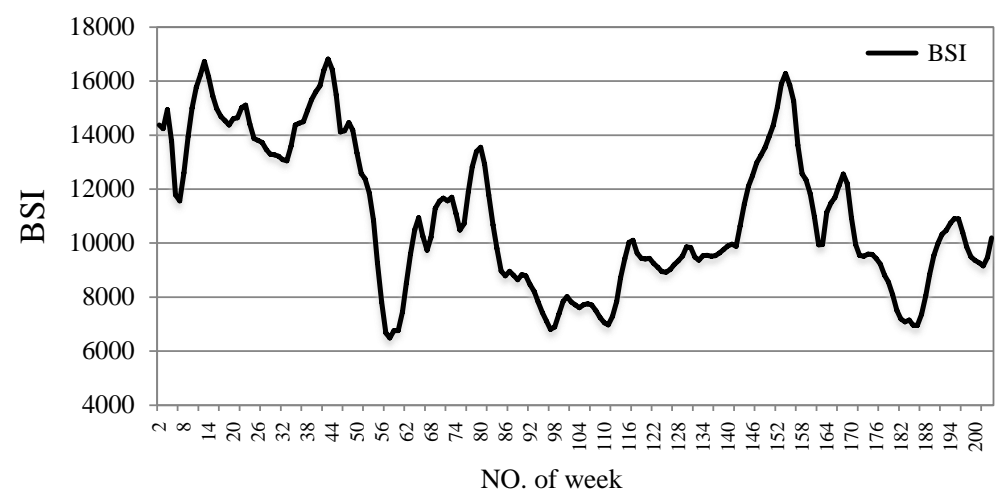

Fig. 6 Historical data of weekly averaged BSI.

\subsection{Database construction}

Before train the SVM model, we need to construct the database which is used for SVM to find the relationship between the history data and the predicted data. The database should be constructed in the form of Tab. I, where $m$ is the dimension of input vectors. By setting different dimensions of input vector, we can construct different training databases. And many research indicate that the predict effect vary with the input dimension.

\begin{tabular}{|c|c|c|c|c|c|}
\hline \multicolumn{5}{|c|}{ Input vector } & $\begin{array}{l}\text { Output vector } \\
\text { BSI }(m+1) \text {, }\end{array}$ \\
\hline $\begin{array}{l}\text { BSI (1), } \\
\text { BSI (1), } \\
\text { BSI (1), } \\
\text { BSI (4), } \\
\text { BSI (5), } \\
\text { BSI (6), }\end{array}$ & $\begin{array}{l}\text { BSI (2), } \\
\text { BSI (2), } \\
\text { BSI (2), } \\
\text { BSI (5), } \\
\text { BSI (6), } \\
\text { BSI (7), }\end{array}$ & $\begin{array}{l}\text { BSI (3), } \\
\text { BSI (3), } \\
\text { BSI (3), } \\
\text { BSI (6), } \\
\text { BSI (7), } \\
\text { BSI (8), }\end{array}$ & $\begin{array}{l}\cdots \\
\cdots \\
\cdots \\
\cdots \\
\cdots \\
\cdots\end{array}$ & $\begin{array}{l}\text { BSI }(m), \\
\text { BSI }(m+1), \\
\text { BSI }(m+2), \\
\text { BSI }(m+3), \\
\text { BSI }(m+4), \\
\text { BSI }(m+5),\end{array}$ & $\begin{array}{l}\text { BSI }(m+1), \\
\text { BSI }(m+2), \\
\text { BSI }(m+3), \\
\text { BSI }(m+4), \\
\text { BSI }(m+5), \\
\text { BSI }(m+6),\end{array}$ \\
\hline
\end{tabular}

Tab. I The constructed database of $m$ dimension input vectors.

\subsection{Model identification}

Firstly, the parameter to be calibrated is the numbers of input vector, which will determine how database is established. The SVM model is used to train and 
to predict the future BSI. This paper takes $70 \%$ of the data in the database as training data, $20 \%$ as test data, and $10 \%$ as inspection data. Besides, MAPE is used to compare the prediction effect under different support vector number cases. Randomly select the sample of training, test and inspection in proportion for ten times to predict, the average result can be seen in Fig. 7.

$$
\mathrm{MAPE}=\frac{1}{K} \sum_{k=1}^{K} \frac{\left|\mathrm{BSI}_{k}-\widehat{\mathrm{BSI}}_{k}\right|}{\mathrm{BSI}_{k}} \times 100 \%
$$

where $K$ is the number of test samples. $\mathrm{BSI}_{k}$ is the observed BSI value of week $k$. $\widehat{\mathrm{BSI}}_{k}$ is the predicted BSI value of week $k$ using the prediction approaches.

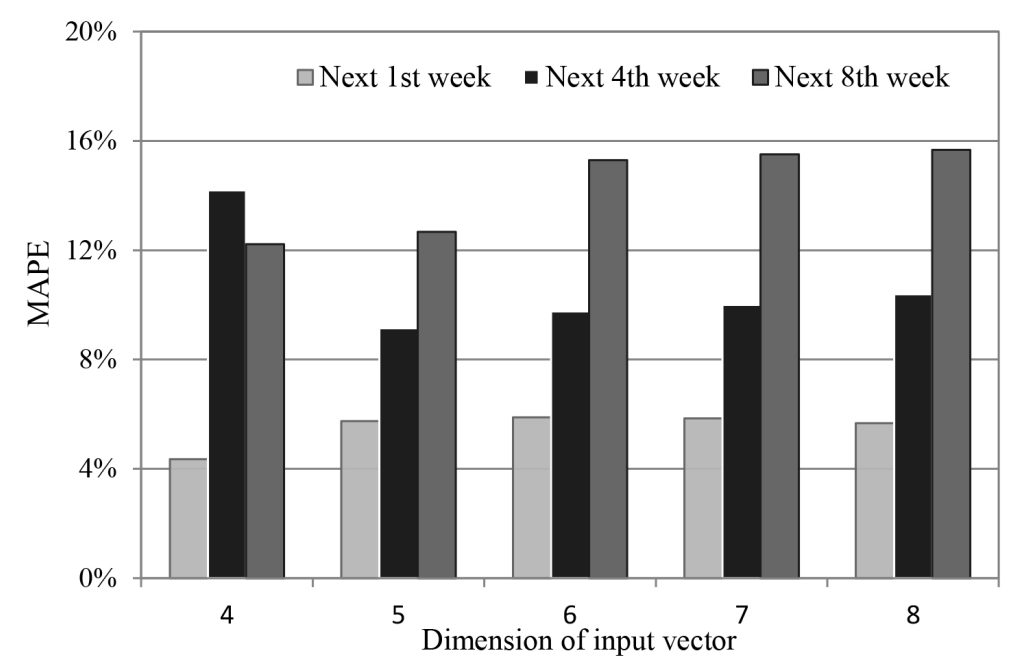

Fig. 7 The performance of model with different dimensions of input vector.

To improve the accuracy of BSI of final week of months, we take next 4,8 week as control point, which could be predicted by one step. As can be seen from the Fig. 7, we use the basic SVM model to predict the BSI of next 1-st week, next 4 -th week, next 8 -th week BSI value. In the term of next $1^{\text {st }}$ week, MAPE is $4.35 \%$ when the input vector dimensions is 4 , while the input dimension is 5 or more than 5, MAPE remain at around 5.80\%. In terms of predicting the following fourth weeks, MAPE reaches $14.19 \%$ when the input dimension is 4 , while the input vector dimension increases from 5 to 8 , MAPE correspondingly increases from $9.15 \%$ to $10.39 \%$. And, MAPE for the forecast of BSI of the next 8-th weeks increase with the expansion of the input dimension. When the input dimension is 4 and 5, MAPE remains at around $12 \%$. For comprehensive consideration, when the input dimension is 5, MAPE is small and keep in the acceptable range, so this article select 5 as input vector dimensions.

In addition, the selection of the kernel function is important when using SVM model to predict BSI. The RBF kernel maps samples into a higher dimensional space and, can handle the case when the relation between class labels and attributes is nonlinear. Furthermore, the linear kernel is a special case of RBF as 
Keerthi and Lin showed: the linear kernel with a penalty parameter $C$ had the same performance as the RBF kernel with some parameters $(C, \varepsilon, \gamma)$. In addition, the number of hyperparameters of the RBF kernel is less than the polynomial kernel, which means the RBF kernel has less numerical difficulties in contrast to polynomial kernels whose values may go to infinity or zero. Moreover, it is noted that the sigmoid kernel is not valid (i.e., not the inner product of two vectors) under some parameters. Therefore, this paper uses the Radial-basis function (RBF) kernel, which has three parameters: $C, \varepsilon$ and $\gamma$. To properly selecting the three parameters, there are several methods developed to identify the best $C$ and $\varepsilon$, among which, grid-search is frequently used as the most reliable but a complex one. In 'grid-search', all pairs of $(C, \varepsilon, \gamma)$ are tried and the one with the best performance is picked up. Therefore, in this paper, the grid-search algorithm is selected to determine the parameters of SVM. In the direct prediction model, the three parameters are selected as $\left(0.01,2^{-4}, 1.56\right)$, while in iterative model, the parameters are selected as $\left(0.01,2^{-4}, 1.83\right)$.

\subsection{Results}

Using the multi-step hybrid prediction model, we can get the prediction result. The weekly BSI value of October 13, 2014 to November 28, 2014, can be seen in Fig. 8.

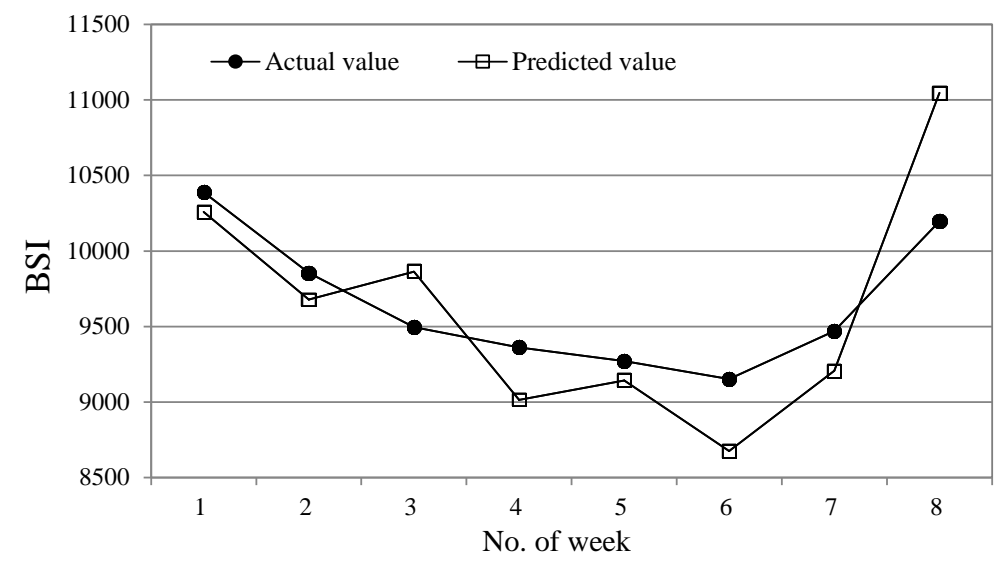

Fig. 8 Predicted result of the testing data.

In order to evaluate the prediction effect of hybrid model, the history mean prediction model (HMP), ARMA model and simple iterative prediction (IP) model are compared.

The history mean prediction model is shown in Eq. (21):

$$
\mathrm{BSI}_{M}=\frac{1}{n} \sum_{i=1}^{n} \mathrm{BSI}_{i}
$$

where $\mathrm{BSI}_{M}$ is the BSI of week $n, \mathrm{BSI}_{i}$ is the BSI of week $i$. The average BSI of the previous $n$ terms is to predict the current data. 
ARMA model is a commonly linear model of limited parameters describing stationary time series, which is suitable for short-term prediction. The predictive model can be seen in Eq. (22). The BSI of each model is obtained after taking the related data into the model as

$$
y_{t}=y_{t-1}+\cdots+\phi_{p} y_{t-p}+\varepsilon_{t}-\theta_{1} \varepsilon_{t-1}-\cdots-\theta_{q} \varepsilon_{t-q}
$$

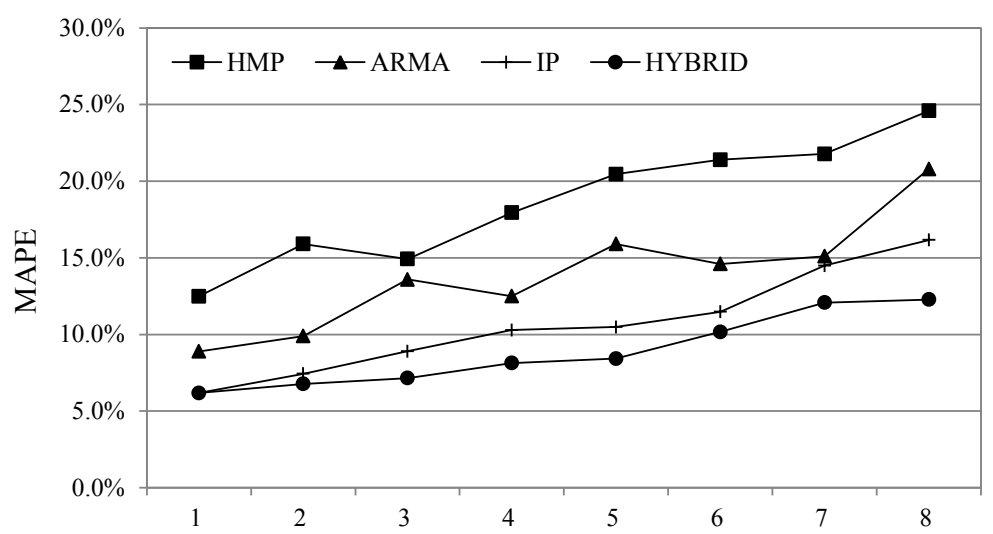

Fig. 9 Comparison of MAPEs of four methods.

From Fig. 9, we can see that the MAPE of all models is added with the increase of week number. And the MAPE of HMP in most case is the largest, which means the performance is the worst. It is because the HMP cannot accurately get the influence of different observed data to predicted data. As for ARMA model, the MAPE of it is less than HMP, while bigger than IP and hybrid prediction model. The MAPE is around 15\%, and the performance is not satisfactory. The MAPE of IP model increase obviously with the prediction step increasing, which is the reason why we propose multi-step hybrid prediction. As Fig. 9 shows, the MAPE of hybrid model is least, and it is less than $15 \%$, which means it has good performance. So, the hybrid model is feasible in multi-step BSI prediction.

\section{Conclusions}

Considering the less reference about freight index of single ship type, this paper intends to predict the BSI based on support vector machine. Predicting BSI value of multi-weeks can provide strong support for company to make decision, which usually can be solved by two methods: direct and iterative prediction. The direct prediction is simple, and the performance is relatively better. However, it requires building database and training the SVM again for predicting BSI of another week. The iterative one can build database and train the SVM only once. However, because of its multi-step prediction, the prediction error is bigger. Therefore, this paper develops a multi-step hybrid prediction model, which combines the direct and iterative model. To evaluate the performance of the hybrid model, we use the weekly averaged BSI value from January, 2011 to November, 2014 as simple data, 
and the performance is compared with other prediction model, such as history mean prediction model, ARMA model and simple iterative prediction model, the result shows that the hybrid multistep prediction model based on SVM has high accuracy, and is feasible in the BSI index prediction.

\section{Acknowledgement}

This work was supported in the special grade of financial support from Higher Education Development Fund (for Collaborative Innovation Center) of Liaoning Province, China 2011011640, the Humanity and Social Science Youth foundation of Ministry of Education of China 13YJCZH042 and Specialized Research Fund for the Doctoral Program of Higher Education of China 20120041120018.

\section{References}

[1] BEENSTOCK M., VERGOTTIS A. Econometric modelling of world shipping. Springer Science \& Business Media, 1993.

[2] BERG-ANDREASSEN J.A. The relationship between period and spot rates in international maritime markets. Maritime Policy and Management. 1997, 24(4), pp. 335-350, doi: 10. 1080/03088839700000042.

[3] CULLINANE K.P.B., MASON K.J., CAPE M. A comparison of models for forecasting the Baltic freight index: Box-Jenkins revisited. Maritime Economics 83 Logistics. 1999, 1(2), pp. 15-39, doi: 10.1057/ijme.1999.10.

[4] FAN Y.H., XING Y.W., YANG H.L. Prediction of Baltic Capesize Freight Index Based on GARCH Model. Applied Mechanics and Materials. 2014, 488, pp. 1494-1497, doi: 10.4028/ wWw.scientific.net/AMM . 488-489.1494.

[5] HAN Q., YAN B., NING G., YU B. Forecasting Dry Bulk Freight Index with Improved SVM. Mathematical Problems in Engineering. 2014, 2014, doi: 10.1155/2014/460684.

[6] HAWDON D. Tanker freight rates in the short and long run. Applied Economics. 1978, 10(3), pp. 203-218,doi: $10.1080 / 758527274$.

[7] KAVUSSANOS M.G., ALIZADEH-M A.H. Seasonality patterns in dry bulk shipping spot and time charter freight rates. Transportation Research Part E: Logistics and Transportation Review. 2001, 37(6), pp. 443-467, doi: 10.1016/S1366-5545(01)00004-7.

[8] KAVUSSANOS M.G., VISVIKIS I.D. Market interactions in returns and volatilities between spot and forward shipping freight markets. Journal of Banking \& Finance. 2004, 28(8), pp. 2015-2049, doi: 10.1016/j.jbankf in.2003.07.004.

[9] KAVUSSANOS M.G. Price risk modelling of different size vessels in the tanker industry using autoregressive conditional heteroskedasticity $(\mathrm{ARCH})$ models. The Logistics and Transportation Review. 1996, 32(2), pp. 161-176.

[10] LEE K.L., BILLINGS S.A. A new direct approach of computing multi-step ahead predictions for non-linear models. International Journal of Control. 2003, 76(8), pp. 810-822, doi: 10 . 1080/0020717031000112276.

[11] LEONOV Y., NIKOLOV V. A wavelet and neural network model for the prediction of dry bulk shipping indices. Maritime Economics 8 Logistics. 2012, 14(3), pp. 319-333, doi: 10. 1057/mel.2012.10.

[12] LI J., PARSONS M.G. Forecasting tanker freight rate using neural networks. Maritime Policy \& Management. 1997, 24(1), pp. 9-30, doi: 10.1080/03088839700000053.

[13] SANSOM D.C., DOWNS T., SAHA T.K. Evaluation of support vector machine based forecasting tool in electricity price forecasting for Australian national electricity market participants. Journal of Electrical and Electronics Engineering. 2003, 22(3), pp. 227-234. 


\section{Neural Network World 3/2016, 219-232}

[14] STRANDENES S.P. NORSHIP: a simulation model for bulk shipping markets. Norwegian School of Economics and Business Administration, 1986.

[15] VAPNIK V.N. The Nature of Statistical Learning Theory. New York: Springer-Verlag, 1995.

[16] VAPNIK V.N. An overview of statistical learning theory. IEEE Transactions on Neural Networks. 1999, 10(5), pp. 988-999, doi: 10.1109/72.788640.

[17] VEENSTRA A.W., FRANSES P.H. A co-integration approach to forecasting freight rates in the dry bulk shipping sector. Transportation Research Part A: policy and practice. 1997, 31(6), pp. 447-458, doi: 10.1016/S0965-8564(97)00002-5.

[18] YANG H., CHAN L., KING I. Support vector machine regression for volatile stock market prediction. In: Proceedings of the 3rd International Conference on Intelligent Data Engineering and Automated Learning - IDEAL, Manchester, UK. 2002, Berlin Heidelberg: Springer, 2002, pp. 391-396, doi: 10.1007/3-540-45675-9_58.

[19] YAO B.Z., YAO J.B., ZHANG M.H., YU L. Improved support vector machine regression in multi-step-ahead prediction for rock displacement surrounding a tunnel. Scientia iranica. Transaction A, Civil Engineering. 2014b, 21(4), pp. 1309-1316.

[20] YAO B.Z., HU P., ZHANG M.H., JIN M.Q. A Support Vector Machine with the Tabu Search Algorithm For Freeway Incident Detection. International Journal of Applied Mathematics and Computer Science. 2014a, 24(2), pp. 397-404, doi: 10.2478/amcs-2014-0030.

[21] YAO B.Z., YU B., GAO J.J., ZHANG M.H. An improved particle swarm optimization for carton heterogeneous vehicle routing problem with a collection depot. Annals of Operations Research. 2014c, pp. 1-18, doi: 10.1007/s10479-015-1792-x.

[22] YU B., YANG Z.Z., CHEN K., YU B. Hybrid model for prediction of bus arrival times at next station. Journal of Advanced Transportation. 2010, 44(3), pp. 193-204.

[23] YU B., SONG X.L., YANG Z.M., YAO B.Z. k-Nearest Neighbor Model for Multiple-TimeStep Prediction of Short-Term Traffic Condition. Journal of Transportation EngineeringASCE. 2015, doi: 10.1061/(ASCE) TE. 1943-5436.0000807.

[24] YU B., KONG L., SUN Y., YAO B.Z., GAO Z.Y. A bi-level programming for bus lane network design. Transportation Research Part C. 2015, 55, pp. 310-327, doi: 10.1016/j. trc.2015.02.014.

[25] YU B., YANG Z., SUN X., YAO B., ZENG Q., Jeppesen E. Parallel genetic algorithm in bus route headway optimization. Applied Soft Computing. 2011, 11(8), pp. 5081-5091, doi: $10.1016 / j$.asoc. 2011.05 .051

[26] ZENG Q., QU C., NG A.K.Y., ZHAO X. A new approach for Baltic Dry Index forecasting based on empirical mode decomposition and neural networks. Maritime Economics 85 Logistics. 2015, doi: 10.1057/mel.2015.2.

[27] ZHANG G., PATUWO B.E., HU M.Y. Forecasting with artificial neural networks: The state of the art. International Journal of Forecasting. 1998, 14, pp. 35-62, doi: 10.1016/ S0169-2070 (97) 00044-7. 